Monatsschr Kinderheilkd 2021 · 169:805-814 https://doi.org/10.1007/s00112-021-01256-z Angenommen: 27. Juni 2021 Online publiziert: 16 . August 2021

(c) Der/die Autor(en) 2021

Redaktion

Wolfgang Sperl, Salzburg

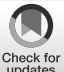

\section{Molekulare Diagnostik und Therapie der Epidermolysis bullosa}

\author{
Multidisziplinär abgestimmte Versorgung in der \\ pädiatrischen Dermatologie
}

\author{
C. Prodinger ${ }^{1,2} \cdot$ A. Klausegger ${ }^{2} \cdot$ J. W. Bauer ${ }^{1,2} \cdot$ M. Laimer $^{1,2}$ \\ ' Universitätsklinik für Dermatologie und Allergologie, Paracelsus Medizinische Privatuniversität Salzburg, \\ Salzburg, Österreich \\ ${ }^{2}$ EB-Haus Austria, Forschungsprogramm für Molekulare Therapie bei Genodermatosen, Universitätsklinik \\ für Dermatologie und Allergologie, Paracelsus Medizinische Privatuniversität Salzburg, Salzburg, \\ Österreich
}

\section{Zusammenfassung}

\section{In diesem Beitrag}

- Grundlagen

- Diagnostik

- Therapeutische Strategien im EB-Haus Austria

Krankheitsmodifizierende Therapien • Korrektive Therapieansätze
Unter Epidermolysis bullosa (EB) subsumiert man genetische Erkrankungen, die durch gesteigerte Hautfragilität mit Blasenbildung nach bereits geringen mechanischen Belastungen gekennzeichnet sind. Hochvariable kutane, extrakutane und Organmanifestationen verursachen eine signifikante Morbidität und Mortalität sowie eine hohe Krankheitslast für Betroffene und Angehörige. Obwohl derzeit nicht heilbar, eröffnen Fortschritte in der molekularen Charakterisierung pathogenetischer Prozesse, in den diagnostischen Techniken und molekularen Therapieansätzen neue Perspektiven. Neben korrektiven, potenziell kurativen Behandlungszugängen mit dem Ziel der Wiederherstellung der Funktion von Gen bzw. Protein stellen krankheitsmodifizierende Strategien eine wertvolle Ergänzung dar. Unter Letztere fallen symptomatische Therapien, die sekundär dysregulierte, den Phänotyp modulierende Entzündungskaskaden adressieren oder zielgerichtete Interventionen hinsichtlich bestimmter Symptome wie Fibrosierung, Juckreiz oder kanzerogener Zelltransformation. Molekulare Verfahren erlauben heute zudem, eine Diagnose und damit Prognose früher und präziser zu stellen, was die genetische Beratung erleichtert. Das Management von EB-Patienten ist komplex und bedarf einer Spezialexpertise und multidisziplinär akkordierten Versorgung. Entsprechende Ressourcen halten designierte Expertisezentren wie das EB-Haus Austria vor, das als Mitglied des Europäischen Referenznetzwerks für Seltene Hauterkrankungen (ERN Skin) sowohl klinische Versorgung, Grundlagen- und klinische Forschungsaktivitäten sowie Zugang zu Aus- und Weiterbildungsprogrammen für Betroffene, Betreuende und medizinisches Fachpersonal gewährleistet.

\section{Schlüsselwörter}

Angeborene Anomalien · Blase · Entzündung · Plattenepithelkarzinom · Gentherapie

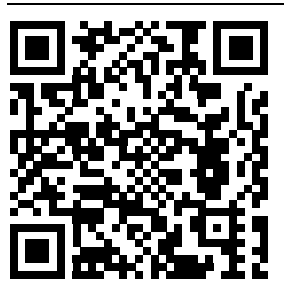

QR-Code scannen \& Beitrag online lesen
Unter dem Begriff Epidermolysis bullosa (EB) subsumiert man eine Gruppe klinisch und genetisch heterogener Genodermatosen mit prototypischer Hyperfragilität und Blasenbildung epithelialisierter Gewebe. In Europa leiden rund 30.000 Menschen (Österreich ca. 300) an dieser Erkrankung. Ein breites Spektrum an mukokutanen, extrakutanen und Or- ganmanifestationen sowie primäre und sekundäre Komplikationen machen die EB zu einer Multisystemerkrankung mit hoher Morbidität, Mortalität sowie psychosozialer und ökonomischer Krankheitslast. Obwohl derzeit nicht heilbar, eröffnen die Fortschritte in der Charakterisierung pathogenetischer Kaskaden der EB und innovative Techniken der mo- 
Tab. 1 Vier Haupttypen der Epidermolysis bullosa ( $E B)$, assoziierte Gene und klinische Charakteristika

\begin{tabular}{|c|c|c|c|c|c|}
\hline $\begin{array}{l}\text { Level der } \\
\text { Blasenbildung }\end{array}$ & EB-Subtyp & Vererbung & Gene & Proteine & Klinische Merkmale \\
\hline \multirow[t]{8}{*}{ Intraepidermal } & \multirow[t]{8}{*}{ EB simplex } & \multirow[t]{3}{*}{$A D$} & $\begin{array}{l}\text { KRT5, } \\
\text { KRT14 }\end{array}$ & Keratin 5, 14 & \multirow{8}{*}{$\begin{array}{l}\text { Häufigste (70\%), mildeste Form } \\
\text { Oberflächliche Blasen/Erosionen } \\
\text { Akral betont, lokalisiert oder generalisiert } \\
\text { Verschlechterung durch Hitze, Schwitzen } \\
\text { Fallweise Schleimhautläsionen, Muskeldystrophie, Kar- } \\
\text { diomyopathie, nephrotisches Syndrom }\end{array}$} \\
\hline & & & PLEC & Plektin & \\
\hline & & & KLHL24 & Kelch-like member 24 & \\
\hline & & \multirow[t]{5}{*}{$A R$} & $\begin{array}{l}\text { KRT5, } \\
\text { KRT14 }\end{array}$ & Keratin 5,14 & \\
\hline & & & DST & $\begin{array}{l}\text { „Bullous pemphigoid } \\
\text { antigen 230“ (BP230) }\end{array}$ & \\
\hline & & & EXPH5 & Exophilin & \\
\hline & & & PLEC & Plektin & \\
\hline & & & CD151 & CD151-Antigen & \\
\hline \multirow[t]{4}{*}{$\begin{array}{l}\text { Junktional/ } \\
\text { Lamina lucida }\end{array}$} & \multirow[t]{4}{*}{$\begin{array}{l}\text { Junktionale } \\
\text { EB }\end{array}$} & \multirow[t]{4}{*}{ AR } & $\begin{array}{l}\text { LAMA3, } \\
\angle A M B 3 \\
\text { LAMC2 }\end{array}$ & Laminin 332 & \multirow{4}{*}{$\begin{array}{l}\text { Blasenbildung, chronische Wunden, atrophe Narbenhei- } \\
\text { lung, Hypergranulation } \\
\text { Nageldystrophie, Zahnschmelzdefekte } \\
\text { Komplikationen (GI-Trakt, Atemwege, Urogenitaltrakt, } \\
\text { Augen) }\end{array}$} \\
\hline & & & COL17A1 & Typ-XVII-Kollagen & \\
\hline & & & $\begin{array}{l}\text { ITGA6, } \\
\text { ITGB4 }\end{array}$ & Integrin $\alpha_{6} \beta_{4}$ & \\
\hline & & & ITGA3 & „Integrin a3 subunit" & \\
\hline \multirow[t]{2}{*}{$\begin{array}{l}\text { Superfizielle } \\
\text { Dermis }\end{array}$} & \multirow[t]{2}{*}{$\begin{array}{l}\text { Dystrophe } \\
\text { EB }\end{array}$} & $A D$ & COL7A1 & Typ-VII-Kollagen & $\begin{array}{l}\text { Blasenbildung mit Narbenabheilung; Milien } \\
\text { Akral betont, lokalisiert } \\
\text { Nageldystrophie; Hyperkeratose an Händen mit Kon- } \\
\text { trakturen } \\
\text { Milder im Vergleich zur rezessiven Form }\end{array}$ \\
\hline & & $A R$ & COL7A1 & Typ-VII-Kollagen & $\begin{array}{l}\text { Chronische Wunden, Narbenbildung, Fibrose, Pseudo- } \\
\text { syndaktylie, ausgeprägter Schleimhautbefall (GI-Trakt, } \\
\text { Atemwege, Urogenitaltrakt, Augen), Nageldystrophie, } \\
\text { Karies, Wachstumsretardierung, schwere Anämie, Osteo- } \\
\text { penie/-porose, Plattenepithelkarzinome }\end{array}$ \\
\hline Gemischt & Kindler-EB & $A R$ & FERMT1 & $\begin{array}{l}\text { "Fermitin family homo- } \\
\log 1 "\end{array}$ & $\begin{array}{l}\text { Akrale Blasenbildung, Fotosensitivität, kutane Atrophie, } \\
\text { progressive Poikilodermie } \\
\text { Ösophageale, urogenitale, gastrointestinale Beteiligung }\end{array}$ \\
\hline
\end{tabular}

lekularen Medizin neue translationale Behandlungsperspektiven für Betroffene.

Die Komplexität der EB erfordert in der Betreuung und Versorgung eine hohe Expertise und eine institutionalisierte interdisziplinäre Zusammenarbeit. Entsprechende Ressourcen bieten designierte Referenzzentren wie das EB-Haus Austria oder EB-Zentrum Freiburg innerhalb des europäischen Referenznetzwerks für seltene Hauterkrankungen (ERN-Skin, [24]). Das EB-Haus Austria wurde 2005 in Salzburg errichtet und vereint die medizinische Versorgung von EB-Patienten, Grundlagenund klinische Forschungsaktivitäten sowie Zugang zu Aus- und Weiterbildungsprogrammen für Betroffene, Betreuende und medizinisches Fachpersonal an einem Standort.

\section{Grundlagen}

Die der EB zugrunde liegenden Mutationen treten in derzeit 16 bekannten Genen auf [14]. Sie führen zum quantitativen (Fehlen/Reduktion) oder qualitativen (Funktionsverlust) Mangel an Proteinen, die maßgeblich für die Aufrechterhaltung der mechanischen und funktionellen Integrität der Epidermis und dermoepidermalen Junktionszone, d.h. der Verbindungszone von Epidermis und darunter liegender Dermis, sind. Klinisch zu beobachten ist eine gesteigerte Verletzlichkeit epithelialisierter Gewebe, die mit der Bildung von Blasen, Erosionen, Ulzera sowie Narben nach bereits geringer mechanischer Belastung einhergeht.

Nach der aktuellen Konsensusklassifikation stellt die EB den Prototyp der Hautfragilitätssyndrome dar, der durch bullö- se Veränderungen primär am Integument gekennzeichnet ist. Abhängig von der Ebene der mutationsbasierten Gewebedehiszenz werden 4 EB-Haupttypen unterschieden; weitere molekulare und klinische Befunde stratifizieren zahlreiche Subtypen (• Tab. 1; [14]). Genotyp-Phänotyp-Korrelationen sind i. Allg. komplex, auch weil neben einer beachtlichen genotypischen $\mathrm{He}$ terogenität epigenetische, biochemische und Umweltfaktoren die phänotypische Ausprägung relevant beeinflussen. Generell lässt sich aber beobachten, dass schwerere Formen mit einem rezessiven Erbgang und biallelischen Komplettverlust der jeweiligen Proteine vergesellschaftet sind $[15,27]$. Hier manifestieren sich die Hautläsionen zumeist generalisiert und bereits bei der Geburt. Die Krankheitslast wiegt oft schwer, ist multidimensional (medizinisch, psychosozial, ökonomisch) und trifft 
Hier steht eine Anzeige.

黑 Springer 

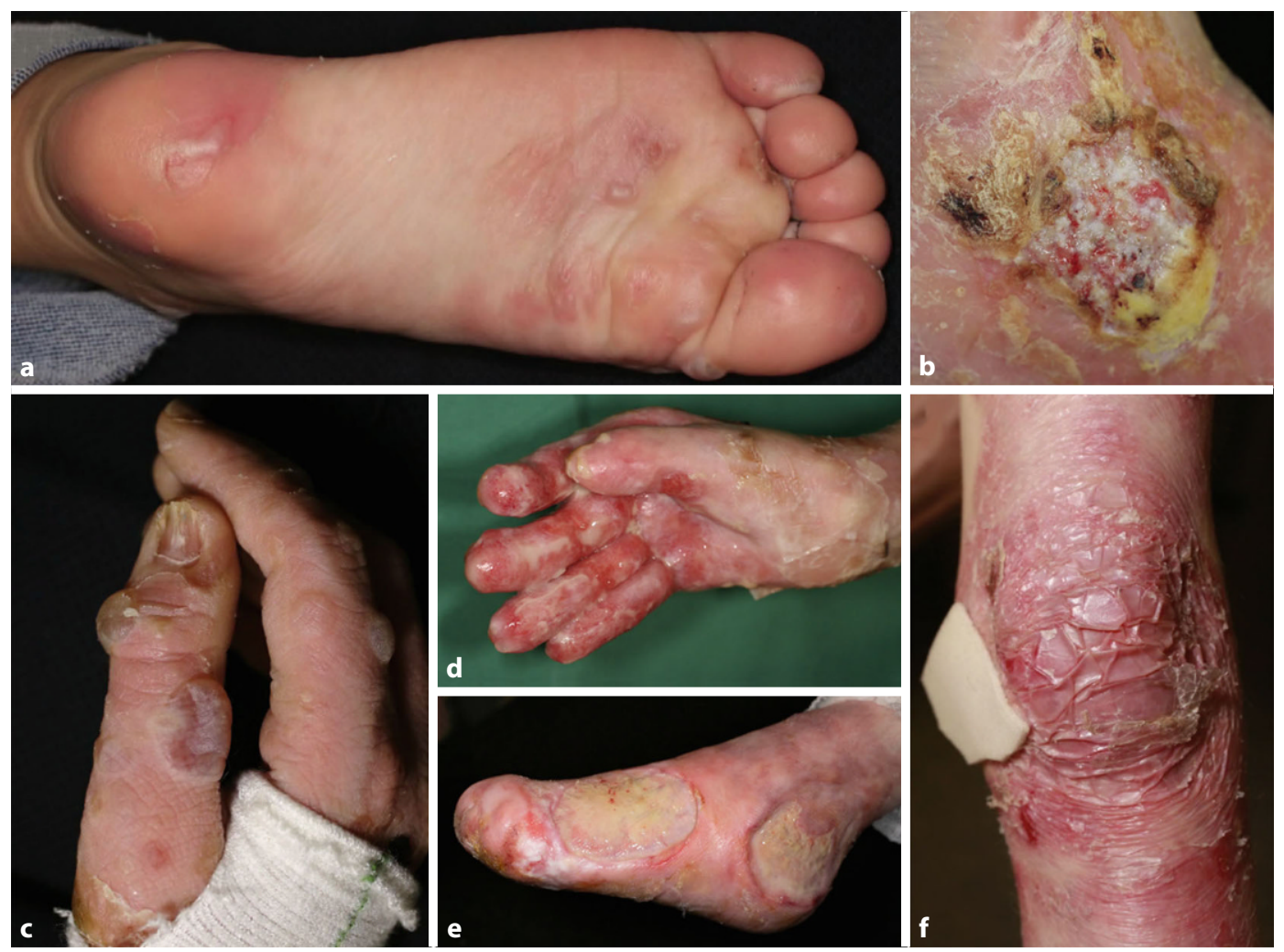

Abb. $1 \Delta$ a Epidermolysis bullosa simplex und vorwiegend auf die Fußsohlen begrenzte Blasenbildung, b junktionale Epidermolysis bullosa (JEB) mit Plattenepithelkarzinom am lateralen Fußrücken, für dessen Entwicklung chronische Wunden prädisponieren, c JEB mit für diese Form typischen prallen Blasen sowie Nageldystrophie, $\mathbf{d}$ chronische Wunden, Erosionen sowie beginnende Pseudosyndaktylie an der Hand eines 6-jährigen Mädchens mit rezessiv dystropher Epidermolysis bullosa (RDEB), e Pseudosyndaktylie mit Verschmelzung und "Cocoon"-artiger Vernarbung der Endglieder neben chronischen, fibrinbelegten Wunden am Fuß bei RDEB. Eine klinische Abgrenzung von reaktiv-hyperplastischen und (prä-)malignen Prozessen ist häufig schwer bis unmöglich.f Atrophe Narben an mechanisch exponiertem Areal (Knie) bei RDEB. (Mit freundl. Genehmigung, $\odot$ R. Hametner, alle Rechte vorbehalten)

PatientInnen und Angehörige. Der lebenslange, meist progressive Verlauf mit Auftreten schwerwiegender Komplikationen in Organsystemen auch jenseits der Haut definiert zusätzlich einen ressourcenintensiven Betreuungs- und Behandlungsaufwand bzw. den Bedarf an effektiveren Therapien ([26]; 0 Abb. 1).

Im Folgenden werden die Prinzipien der molekularen Diagnostik und Therapie anhand des Portfolios des EB-Haus Austria dargestellt.

\section{Diagnostik}

Eine prognostisch relevante, damit frühzeitig indizierte (Sub-)Typisierung im Neugeborenen- bzw. frühen Kindesalter einzig auf Basis klinischer Merkmale ist aufgrund zunächst überlappender Phänotypen schwierig bis unmöglich. Für die Diagnosepräzisierung ist daher eine (periläsionale) Hautbiopsie mit Immunfluoreszenzfärbung und eine genetische Analyse unumgänglich. Bei speziellen Fragestellungen ergänzt ggf. die morphologische Charakterisierung von Strukturkomponenten der Basalmembranzone mittels Elektronenmikroskopie den diagnostischen Algorithmus [25].

\section{॥ Eine Hautbiopsie mit Immunfluoreszenzfärbung und eine genetische Analyse sind unumgänglich}

Mithilfe der Immunfluoreszenzfärbung werden die Ebene der Spaltbildung (Nachweis des Referenzproteins am Blasendach oder am Blasenboden) sowie die Lokalisation und semiquantitativ der Grad der Expression von Indexproteinen bestimmt. Hierzu finden spezifische Färbeantikörper gegen Strukturproteine der Haut oder der Basalmembranzone Anwendung. Die Technik erlaubt, sehr rasch und mit wenig Aufwand wichtige Hinweise auf den vorliegenden Subtyp bzw. das betroffene Gen zu erlangen [18]. Der Befund ist direktiv für den weiteren, molekularen Analysegang, dessen Standardisierbarkeit für Registerdaten, Präzision, Breitspektrum („nextgeneration/panel sequencing"), diagnostische und therapeutische Implikationen, breite Verfügbarkeit und immer günstigere Kosten den heutigen Goldstandard definieren ( $\bullet \mathbf{A b b}$. 2). Mittlerweile können $90 \%$ aller EB-Verdachtsfälle molekular eindeutig diagnostiziert werden $[15,16]$.

\section{Therapeutische Strategien im EB- Haus Austria}

Da bislang kurative Therapien nicht verfügbar sind, beruht die Behandlung von PatientInnen auf der Vermeidung von 


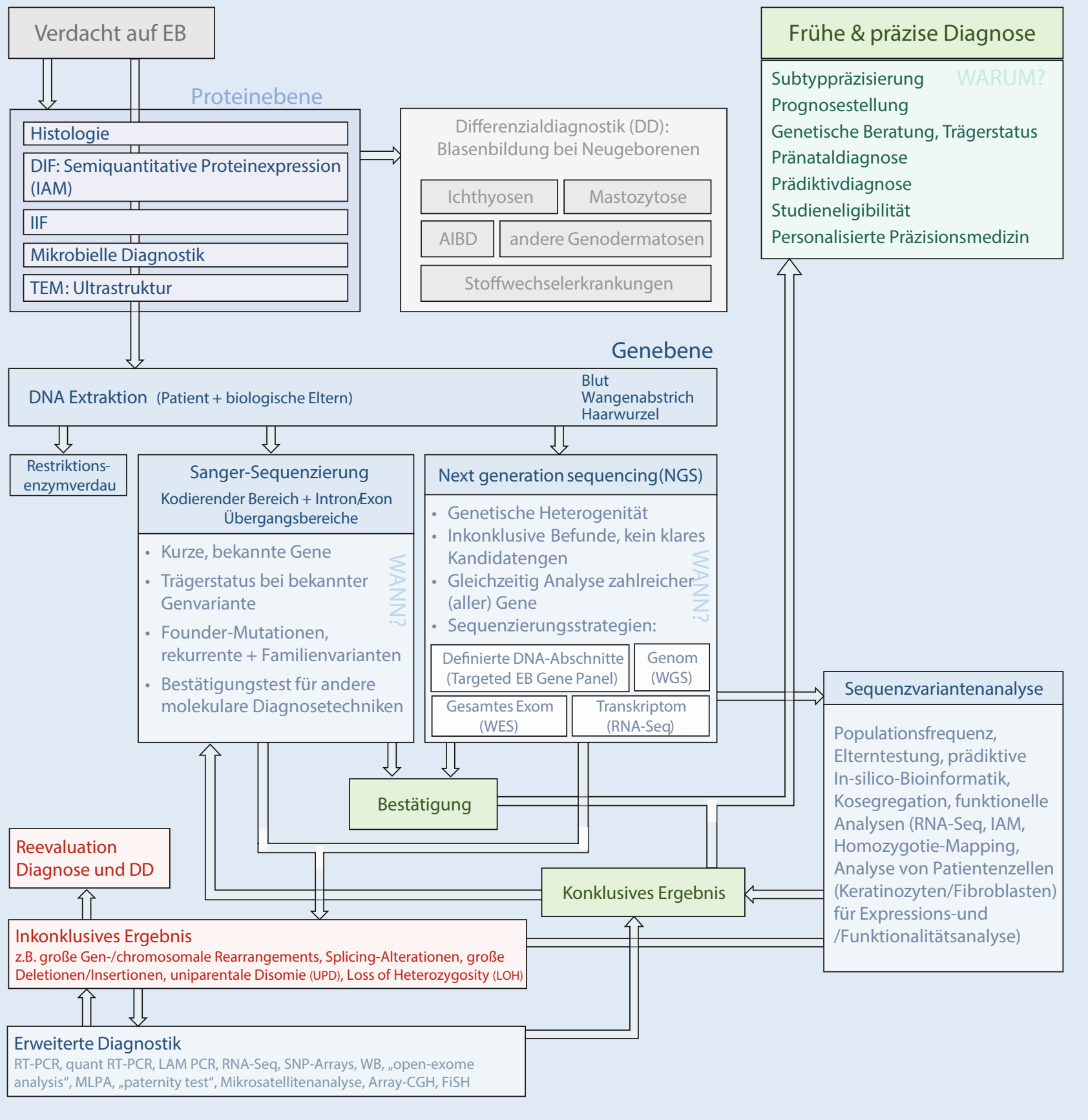

Abb. 2 \ Diagnostischer Algorithmus, gültig für alle Subtypen der Epidermolysis bullosa (EB).DIF direkte Immunfluoreszenz, IIF indirekte Immunfluoreszenz, TEM Transmissionselektronenmikroskopie, WGS "whole genome sequencing", RNASeq RNA-Sequenzierung, WES "whole exome sequencing", IAM "immunofluorescence antigen mapping", $R T$ - $P C R$ "real-time polymerase chain reaction", quant $R T-P C R$ quantitative RT-RCP, $L A M P C R$ "linear-amplification mediated PCR", SNP "single nucleotide polymorphism", WB Western-Blot, MLPA "multiplex ligation-dependent probe amplification", Array-CGH "arraybased comparative genomic hybridization“. (Mit freundl. Genehmigung, $\odot$ C. Prodinger, alle Rechte vorbehalten)

Provokationsfaktoren (wie Trauma, Hitze) und symptomatisch-supportiven Maßnahmen. Dazu zählen Prävention und Behandlung von Wunden, mikrobiellen Infektionen, Schmerzen, Juckreiz und
Komplikationen wie Anämie, Malnutrition oder Plattenepithelkarzinomen.

Neue Erkenntnisse zur molekularen Pathogenese, innovative molekularbiologische Techniken und Methoden, spezifische Forschungsförderprogramme und
Zulassungserleichterungen auf dem Gebiet der seltenen Erkrankungen („orphan drug legislature ") befeuerten in den letzten Jahren translationale Forschungsinitiativen. Mittlerweile sind dadurch auch zahlreiche, vielversprechende Therapiean- 
sätze in der klinischen Testung angelangt. Hierbei sind zwei prinzipielle Strategien zu unterscheiden: einerseits korrektive, potenziell kurative Verfahren, die zum Ziel haben, den genetischen Defekt dauerhaft zu korrigieren; und andererseits krankheitsmodifizierende Interventionen, die insbesondere auf sekundär entzündliche Prozesse wirken, die als Folge der mutationsbasiert-permanenten Gewebezerstörung unproduktiv chronifizieren und einen beachtlichen Kollateral- und additiven Langzeitschaden verursachen. So kommen Strategien der Gen-, Protein-, RNA- und zellbasierten Therapien ebenso zum Einsatz wie "small molecules" oder "drug repurposing", d.h. die Anwendung bereits verfügbarer Wirkstoffe in einem neuen Kontext, dem der EB [23].

\section{Krankheitsmodifizierende Therapien}

\section{Inflammation als therapeutisches}

Ziel

Neben dem primär strukturell-funktionellen Defekt wird vermehrt die Bedeutung des dadurch unterhaltenen, chronischen Gewebsschadens mit Induktion und Dysregulation von inflammatorischen Signalkaskaden als pathogenetisch relevanter Sekundärmechanismus bei EB erkannt. Vor allem bei den schweren Subtypen führt der exzessive, generalisierte Hautbefall (z.B. rezessiv dystrophe Epidermolysis bullosa, RDEB) nicht nur läsional begrenzt zu einem entzündlich alterierten Mikromilieu, sondern ähnlich wie bei schwerer atopischer Dermatitis oder Psoriasis auch zur systemischen Streuung von Entzündungsmediatoren. Die bei EB gestörte Hautbarrierefunktion begünstigt u.a. zusätzlich durch eine transkutane Invasion von Mikroben den Erhalt dieser kontraproduktiven proinflammatorischen Reaktionen; diese hemmen die Wundheilung, fördern eine Dysbiose und initiieren einen kanzerogenen Gewebsumbau („tissue remodeling“ mit Aktivierung proliferativer Signalwege). Die Entzündungsreaktionen verstärken auch die oft quälende Juckreizempfindung der PatientInnen, die die Barrierestörung dann durch Manipulation (Kratzen) verstärken.

Diese an geläufigere Hauterkrankungen mit Barrierestörung erinnernden entzündlichen Mechanismen eröffnen gleichsam die Perspektive neuer Therapieansät- ze, die auf Immunmodulation, verbesserte Wundheilung, Barriererekonstitution, Infektionskontrolle und Hemmung kanzerogener Gewebeprozesse abzielen.

\section{I) Entzündliche Mechanismen eröffnen die Perspektive neuer Therapieansätze}

Beispielsweise wurde Diacerein, ein aus der Rhabarberpflanze extrahiertes Anthranoid, ursprünglich zugelassen zur Behandlung von degenerativen Gelenkerkrankungen (Arthrose), als 1\%ige Creme zur Hemmung von Entzündungsprozessen bei EBS eingesetzt. Dabei zeigten Vordaten, dass EB-simplex-Mutationen in den Genen KRT5 und KRT14 die Aggregation und Destruktion des epidermalen intermediären Filamentnetzwerks führen, was mit verstärkter Zytolyse von Keratinozyten und folglich verstärkter Expression des proinflammatorischen Zytokins IL-1 $1_{\beta}$ einhergeht. Letzteres bewirkt eine Aktivierung des "c-Jun N-terminal-kinase"(JNK)Stresssignalwegs, der seinerseits eine verstärkte Zellapoptose vermittelt. Zusätzlich kommt es über eine positive FeedbackSchleife zur weiteren IL- $1_{\beta}$-Erhöhung. Die klinische Effektivität (Reduktion von Blasen) und Verträglichkeit von topischem Diacerein bei EB-PatientInnen wurde bereits in klinischen Phase-II-Studien nachgewiesen; weitere sind in Planung [1, 28, 29].

Auch topisches Calcipotriol, ein aktives Vitamin- $D_{3}$-Analog, das bei Psoriasis breiten Einsatz findet, hat immunmodulatorische Wirkung, begünstigt die Expression von antimikrobiellen Peptiden, insbesondere Cathelicidin, und beeinflusst das inerte und das adaptive Immunsystem sowie die Proliferation und Differenzierung von Keratinozyten. Diese Eigenschaften haben sich auch bei Patienten mit dystropher Epidermolysis bullosa (DEB), die häufig unter einem Vitamin-D-Mangel leiden, als therapeutisch wirksam erwiesen. Niedrig dosiertes Calcipotriol $(0,05 \mathrm{mg} / \mathrm{g}$ in einer ambiphilen Salbe [Ultraphil ${ }^{\circledR}$ ]) führte in einer Phase-II-Studie bei Patienten mit RDEB zu einer erhöhten mikrobiellen Abwehr, Juckreizreduktion und beschleunigten Wundheilungsförderung [13].

Diese krankheitsmodifizierenden Therapieansätze kennzeichnen sich durch eine im Vergleich zu den mutationsspezifischen molekularen Techniken weniger präzise Wirkung. Es ist unklar, welche Determinanten mit welcher Dynamik das Entzündungsprofil inter- und intraindividuell bestimmen. So können mutationsspezifische molekulare Aberrationen, das für die anatomische Region spezifische Mikromilieu, die (lokale) mikrobielle Flora, Vortherapien und vieles mehr die Entzündungssignaturen modifizieren. Es bleibt also abzuwarten, inwiefern es gelingt, ein Leitzytokin als universell(er)en therapeutischen Ansatzpunkt zu definieren, und inwiefern ggf. verstärkt personalisierte Zugänge sicher umsetzbar sind und hinsichtlich ihrer Wirksamkeit nicht an redundanten Signalkaskaden oder Umgehungskreisläufen scheitern.

\section{Gezielte Therapieansätze für} spezielle EB-Manifestationen

Plattenepithelkarzinome. Plattenepithelkarzinome (PLECA) stellen eine schwerwiegende Komplikation insbesondere bei RDEB dar. Sie treten häufig multipel und bereits im frühen Erwachsenenalter auf und nehmen einen hochaggressiven Verlauf. Die Prognose ist schlecht: Die Mortalität beträgt bis zum 40. Lebensjahr $84 \%$. Das RDEB-assoziierte PLECA ist damit die häufigste Todesursache in dieser Patientenkohorte.

\section{॥ Die EB-Plattenepithelkarzinome nehmen einen aggressiven Verlauf mit schlechter Prognose}

Die Tumoren treten v.a. in exponierten Arealen mit chronischen Wunden und Narben auf. Eine frühe Diagnose wird durch überlagerte Entzündungsprozesse mit reaktiven Gewebehyperplasien und starken Krustenauflagerungen erschwert.

Als zugrunde liegende Pathomechanismen werden u.a. ein repetitiver Gewebestress und damit bedingte Gewebeumbauprozesse diskutiert. Sie führen zu einem abgeänderten Genexpressionsprofil von Fibroblasten, einer verstärkten fibrotischen Umwandlung des Bindegewebes (Matrixversteifung), einer folglich veränderten mechanobiochemischen Signaltransduktion (mit verstärkter Telomeraseaktivität und Apoptoseresistenz bei reduzierter Expression von Tumor- 
Hier steht eine Anzeige.

黑 Springer 
suppressorgenen) und einer forcierten epithelial-mesenchymalen Transition in Keratinozyten. Letzteres geht u.a. mit einem Polarisationsverlust und verstärkter Migrationstendenz dieser Zellen einher. Insgesamt wird ein proentzündliches, kanzerogenes Mikromilieu gefördert [7].

Um die therapeutisch-prognostisch relevante Unterscheidbarkeit von reaktiven, prämalignen und malignen Gewebeveränderungen in chronischen EBWunden zu verbessern, wird derzeit versucht, aus Wundabstrichen gewonnene MicroRNA(miRNA) Profile zu validieren. Präklinisch konnte bereits demonstriert werden, dass aus den extrazellulären Vesikeln von RDEB-Tumorzellen isolierte miRNA ein im Vergleich zu normalen Keratinozyten ein tumorspezifisches Transkript aufweisen. Zudem haben miRNA perspektivisch das Potenzial, als Substrat in einer Flüssigbiopsie auch als Tumormarker im Blut zu fungieren $[27,30]$.

Die EB-PLECA sprechen schlecht auf konventionelle Chemo- und Radiotherapien an. Angesichts der hohen Letalität sind effektive Therapien daher dringend notwendig. Als ein möglicher spezifischer Ansatz zeigte der "Polo-like-kinase 1"(PLK1)Inhibitor Rigosertib im Tiermodell eine selektive Wirkung auf RDEB-PLECA-Tumorzellen und reduzierte das Tumorwachstum [3]. Die Wirksamkeit und Sicherheit von Rigosertib werden derzeit bei PatientInnen mit lokal-fortgeschrittenem oder metastasiertem PLECA in einer Studie getestet (NCT03786237). Zudem wurden anekdotisch die Anwendung der bereits bei anderen Tumoren und Nicht-EB-PLECA erfolgreich verabreichten Checkpoint-Inhibitoren berichtet [10]. Umfassendere Daten zu Sicherheit und Wirksamkeit dieser Medikamente bei EB-Patientinnen sind allerdings wünschenswert, da die typischen autoimmunvermittelten Nebenwirkungen dieser Substanzklassen v.a. an der Haut und im Gastrointestinaltrakt für EB-PatientInnen mit mukokutanen Läsionen, Dystrophie und fortgeschrittenem Tumorstadium kritisch-limitierend sein könnten. Zudem ist nicht ausgeschlossen, dass die chronischen Entzündungsprozesse, die bei EB beschrieben sind, die Wirksamkeit der Immuncheckpoint-Inhibitoren nachteilig beeinflussen könnten.

\section{Korrektive Therapieansätze}

Korrektive, damit potenziell kurative Methoden zielen auf eine nachhaltige bis permanente Restitution der funktionalen Proteinexpression ab. Zu diesem Zweck erfolgen korrigierende Eingriffe aufDNA-, RNA-, mRNA-, Protein- oder Zellebene und zwar entweder in vivo oder ex vivo.

\section{RNA-basierte (genetische)} Therapien

Die meisten Exons bei junktionaler Epidermolysis bullosa (JEB) und DEB treten „in frame“ auf, sodass beim etwaigen Verlust eines einzelnen Exons der Leserahmen des Gens und damit die Proteinfunktion zumeist aufrechterhalten bleiben [17]. Auf dieser Tatsache beruht die Methode des „exon-skipping" durch „Antisense“-Oligonukleotide (AON). Antisense-Oligonukleotide sind kleine Abschnitte modifizierter RNA, die das Überspringen von mutationstragenden Exons während des Transkriptionsprozesses bewirken. Das resultierende Protein ist dann zwar leicht verkürzt, in seiner Funktion aber nicht kompromittiert [6].

Bereits in einer klinischen Studie bei RDEB-Patienten wird das AON QR-313 gegen das mutationstragende Exon 73 des COL7A1-Gens untersucht [5]. Erst kürzlich konnte im Labor des EB-Haus Austria eine effektive topische Anwendung von AON exon-skipping in einem 3D-Hautmodell für JEB (Exon 7 des COL17A1-Gens) demonstriert werden. Als Trägersubstanz der AON fungierten eigens für diese Methode konstruierte Liposomen, deren Hüllenaufbau einer Zellmembran ähnelt und die mit Zielzellen fusionieren [2].

Eine präklinische Genkorrektur auf (Pre-)mRNA-Ebene erlaubt zudem die Technik des "trans-splicing" („spliceosome-mediated RNA trans-splicing", SMaRT). Mithilfe eines Reparaturmoleküls (RTM) wird der fehlerhafte endogene Pre-mRNAAbschnitt während des In-trans-splicingVorgangs durch eine exogene, korrigierte RNA-Version ersetzt. In vitro gelang bereits der Nachweis von Genkorrekturen bei Mutationen in den Genen PLEC, KRT14, COL7A1 und COL17A1. Bislang fanden überwiegend virale Vektoren zur Einschleusung der Moleküle in die Zielzellen Verwendung. Diese Vektoren sind allerdings mit dem Risiko behaftet, eine Insertionsmutagenese $\mathrm{zu}$ verursachen. Darunter versteht man die ungerichtete Insertion eines (korrektiven) DNA-Fragments in das Genom, was Genfunktionen nachteilig verändern bzw. ausschalten kann [6, 22].

Nichtvirale Vektoren bieten in diesem Zusammenhang eine höhere Sicherheit. Kürzlich gelang unter Verwendung eines „minicircle plasmid“ (als RTM-Träger), das per Luftdruck (mithilfe einer "gene gun") in die relevante Hautebene eingebracht wurde, der Effektivitätsnachweis in einem artifiziell generierten Hautmodell. Technische Optimierungen hinsichtlich der Reduktion möglicher "Off-target"-Effekte, einer Effizienzsteigerung des Trans-splicingProzesses sowie des stabilen Transfers des Korrekturkonstrukts in die Haut und Zielzellen werden aktuell durchgeführt, um diese Methode einer klinischen Anwendbarkeit näher zu bringen [22].

\section{DNA-basierte (genetische) \\ Therapien}

Ex-vivo-Genersatz. Bei der Genersatztherapie wird das dysfunktionale Gen mit einer synthetischen Kopie eines funktionierenden (korrigierenden) Gens ersetzt. Der "Complementary-DNA“(cDNA)-Transfer auf Patientenzellen, die mithilfe einer Hautbiopsie gewonnen werden, erfolgt aus technischen Gründen in vitro, d.h. ex vivo. Als Vektoren dienen häufig $\gamma$-Retroviren oder Lentiviren, die eine relativ hohe Transduktionseffizienz aufweisen. Die korrigierten Zellen werden anschließend zu (epidermalen) Hauttransplantaten kultiviert und danach auf die Wundareale des Patienten transplantiert.

Die Ex-vivo-Gentransfer-Methode wurde erstmals 2006 erfolgreich bei einem JEBPatienten mit $L A M B 3-M u t a t i o n e n$ durchgeführt. Diese Transplantate erweisen sich noch heute als biomechanisch stabil. Zwei weitere JEB-Patienten wurden in Folge mit der gleichen Technik behandelt. Einer davon war ein 7-jähriger Junge, dem 2015 nach großflächiger Hautablösung im Zusammenhang mit einer bakteriellen Infektion $80 \%$ des Integuments mit Transplantaten korrigierter Haut ersetzt wurden.

In Folgestudien konnte bei diesem Patienten gezeigt werden, dass die Langzeiteffektivität vom Vorhandensein erfolg- 
Infobox 1

EB-Haus Austria

- https://www.eb-haus.org/

reich transduzierter "Holoclones" in den Transplantaten abhängig ist. Holoclones sind langlebige epidermale Stammzellen, die volle Regenerationskapazität besitzen und somit zur dauerhaften/ kontinuierlichen Erneuerung der Epidermis befähigt sind $[4,8,19]$. Phase-l-/ Phase-II-Folgestudien zur Evaluierung der Sicherheit und Effektivität von autologen epidermalen Stammzelltransplantationen für RDEB- und JEB-Patienten mit COL7A1- und COL17A1-Mutationen laufen (NCT02984085, NCT03490331).

\section{॥ Langzeiteffektivität der Ex- vivo-Gentherapie hängt von der Rate erfolgreich transduzierter Holoclones ab}

Ein anderes Forscherkonsortium berichtete Ergebnisse zur Wirksamkeit von autologen, genkorrigierten Keratinozytentransplantaten bei 7 RDEB-Patienten. Hier erfolgte die Korrektur ohne Identifikation von Stammzellen mithilfe eines COL7A1cDNA-tragenden $\gamma$-Retrovirus. Nichtsdestotrotz konnten selbst 2 Jahre nach der Behandlung eine verbesserte Wundheilung im Indexareal im Vergleich zu Placebo und bei 2 behandelten Patienten auch eine erhöhte Typ-VII-Kollagen-Expression nachgewiesen werden, während schwerwiegende Nebenwirkungen ausblieben [11].

In einer weiteren, derzeit laufenden Studie für RDEB-Patienten werden sowohl autologe Keratinozyten als auch Fibroblasten mit einem selbstinaktivierenden retroviralen Vektor transduziert. Hintergrund für diesen Ansatz sind Labordaten, die darauf hinweisen, dass das Zusammenspiel dieser beiden Zellen als jeweils eigenständige Produzenten von Typ-VII-Kollagen für die regelrechte Bildung von Ankerfibrillen in der Basalmembranzone notwendig zu sein scheint (NCT04186650, [12]).

Technologische Herausforderungen der Ex-vivo-Strategien limitieren derzeit ihre klinische Anwendung. Insbesondere Vektorensicherheit (Insertionsmutagenese), Schwierigkeiten des zielgerichteten Transfers großer Gene wie COL7A1, die
Identifizierung und effiziente Transduktion von Holoclones, potenzielle Autoimmunphänomene gegen therapeutisch induzierte Neoantigene oder auch die Invasivität und damit Belastung des operativen Eingriffs bei hochvulnerablen PatientInnen bedürfen der Beachtung und methodischen Weiterentwicklung [9, 23].

Ex-vivo-Geneditierung. Auf noch präklinischer Ebene werden Methoden der Gen-Editierung mithilfe des CRISPR/Cas9Systems (CRISPR: „clustered regularly interspaced short palindromic repeats"), der Zinkfinger-Nukleasen oder der „transcription activator-like effector nuclease" (TALEN) vorangetrieben, um eine permanente Genkorrektur auf DNA-Ebene zu erreichen. Einer Forschungsgruppe des EBHaus Austria gelang bereits die Korrektur einer Mutation im Exon 73 des COL7A1Gens in RDEB-Keratinozyten mithilfe des CRISPR/Cas9-Systems. Letzteres induziert einen DNA-Doppelstrangbruch in der Nähe der Mutation, der anschließend über zelleigene Mechanismen repariert wird. Dabei kommt es zu einer präzisen Nukleotidinsertion und Wiederherstellung des Leserahmens des Gens. Die so korrigierten Zellen könnten schließlich für die Herstellung von genmodifizierten Hautäquivalenten verwendet werden, um sie auf PatientInnen zu transplantieren. Die klinische Umsetzung wird aktuell noch durch Sicherheitsbedenken, v.a. im Hinblick auf Off-target- und Langzeiteffekte bzw. Immunreaktion gegenüber editierten (korrigierten) Proteinen oder Bestandteilen des CRISPR-Komplexes, gehemmt. Zudem sind auch hier, analog zur Genersatztherapie, invasive Eingriffe notwendig, um die Korrektursysteme in die Haut zu transferieren (Mikrochirurgie, Genkanone, [20, 21]).

\section{Fazit für die Praxis}

- Epidermolysis bullosa (EB) umfasst eine heterogene Gruppe von blasenbildenden Erkrankungen, von denen sich v.a. schwere Verlaufsformen zu Multisystemerkrankungen entwickeln und mit hoher Morbidität einhergehen.

- Pathogenetisch relevante, durch den Strukturschaden initiierte chronische Entzündungsreaktionen begünstigen Infektionen, Dystrophie und die Entwicklung aggressiver Plattenepithelkarzinome. Letztere stellen bei der rezessiv dystrophen Epidermolysis bullosa (RDEB) die häufigste Todesursache dar.

- Die rasche, präzise Diagnosestellung und eine individualisierte, multidisziplinär-akkordierte Betreuung der Patienten sind Qualitätskriterien der Versorgung Betroffener.

- Die EB ist nicht heilbar. Die Entwicklung molekular-korrektiver Therapieansätze ist erfreulich, ihre Umsetzbarkeit im medizinischen Alltag derzeit noch stark limitiert. Pathogenetisch-plausible, krankheitsmodifizierende und immunmodulatorische Behandlungsansätze gewinnen in der Zwischenzeit als ergänzende palliative Strategien an Bedeutung, um die Lebensqualität Betroffener zu verbessern.

\section{Korrespondenzadresse}

\section{Univ.-Prof. Dr. M. Laimer}

Universitätsklinik für Dermatologie und Allergologie, Paracelsus Medizinische

Privatuniversität Salzburg

Müllner Hauptstr. 48, 5020 Salzburg, Österreich m.laimer@salk.at

Funding. Open access funding provided by Paracelsus Medical University.

\section{Einhaltung ethischer Richtlinien}

Interessenkonflikt. M. Laimer gibt an: Grant/ Forschung/klinische Studien, Support: Bristol-Myers Squibb (Plattenepithelkarzinom), Onconova Therapeutics, Amryt Pharma, Rheacell, Holostem Terapie Avanzate, Castle Creek Pharmaceuticals. J.W. Bauer gibt an: Grant/Forschung/klinische Studien, Support: Onconova Therapeutics, Amryt Pharma, Rheacell, Holostem Terapie Avanzate, Castle Creek Pharmaceuticals; Anteilseigner:Diaderm Ges.m.b.H:Kommerzialisierung von diacereinhaltiger $\mathrm{Creme}$. C. Prodinger und A. Klausegger geben an, dass kein Interessenkonflikt besteht.

Für diesen Beitrag wurden von den Autoren keine Studien an Menschen oder Tieren durchgeführt. Für die aufgeführten Studien gelten die jeweils dort angegebenen ethischen Richtlinien.

Open Access. Dieser Artikel wird unter der Creative Commons Namensnennung 4.0 International Lizenz veröffentlicht, welche die Nutzung, Vervielfältigung, Bearbeitung, Verbreitung und Wiedergabe in jeglichem Medium und Format erlaubt, sofern Sie den/die ursprünglichen Autor(en) und die Quelle ordnungsgemäß nennen, einen Link zur Creative Commons Lizenz beifügen und angeben, ob Änderungen vorgenommen wurden.

Die in diesem Artikel enthaltenen Bilder und sonstiges Drittmaterial unterliegen ebenfalls der genannten Creative Commons Lizenz, sofern sich aus der Abbildungslegende nichts anderes ergibt. Sofern das be- 
treffende Material nicht unter der genannten Creative Commons Lizenz steht und die betreffende Handlung nicht nach gesetzlichen Vorschriften erlaubt ist, ist für die oben aufgeführten Weiterverwendungen des Materials die Einwilligung des jeweiligen Rechteinhabers einzuholen.

Weitere Details zur Lizenz entnehmen Sie bitte der Lizenzinformation auf http://creativecommons.org/ licenses/by/4.0/deed.de.

\section{Literatur}

1. Ablinger M, Felder TK, Wimmer M et al (2018) Basal pharmacokinetic parameters of topically applied diacerein in pediatric patients with generalized severe epidermolysis bullosa simplex. Orphanet J Rare Dis 13:193

2. Ablinger M, Lettner T, FriedI N et al (2021) Personalized development of antisense oligonucleotides for Exon skipping restores type XVII collagen expression in junctional epidermolysis bullosa. Int J Mol Sci 22(7):3326

3. Atanasova VS, Pourreyron C, Farshchian M et al (2019) Identification of Rigosertib for the treatment of recessive dystrophic epidermolysis bullosa-associated squamous cell carcinoma. Clin Cancer Res 25:3384-3391

4. Bauer JW, Koller J, Murauer EM et al (2017) Closure of a large chronic wound through transplantation of gene-corrected epidermal stem cells. J Invest Dermatol 137:778-781

5. Bornert O, Hogervorst M, Nauroy P et al (2021) QR-313, an antisense oligonucleotide, shows therapeutic efficacy for treatment of dominant and recessive dystrophic epidermolysis bullosa: a preclinical study. J Invest Dermatol 141(4):883-893.e6

6. Bornert O, Peking P, Bremer J et al (2017) RNA-based therapies for genodermatoses. Exp Dermatol 26:3-10

7. Condorelli AG, Dellambra E (2019) Epidermolysis bullosa-associated squamous cell carcinoma: from pathogenesis to therapeutic perspectives. Int J Mol Sci 20(22):5707

8. De Rosa L, Carulli S, Cocchiarella Fetal (2014) Longterm stability and safety of transgenic cultured epidermal stem cells in gene therapy of junctional epidermolysis bullosa. Stem Cell Rep 2:1-8

9. De Rosa L, Latella MC, Secone Seconetti A et al (2020) Toward combined cell and gene therapy for genodermatoses. Cold Spring Harb Perspect Biol 12:(5):a035667

10. Duong T, Wong D, Barrett A et al (2021) Successful use of immunotherapy to treat advanced cutaneous squamous cell carcinoma in recessive dystrophic epidermolysis bullosa. BMJ Case Rep 14:(2):3238966

11. Eichstadt S, Barriga M, Ponakala A etal (2019) Phase $1 / 2$ a clinical trial of gene-corrected autologous cell therapy for recessive dystrophic epidermolysis bullosa. JCI Insight 4(19):e130554

12. Gaucher S, Lwin SM (2020) EBGene trial: patient preselection outcomes for the European GENEGRAFT ex vivo phase I/II gene therapy trial for recessive dystrophic epidermolysis bullosa. $\mathrm{Br} \mathrm{J}$ Dermatol 182:794-797

13. Guttmann-Gruber C, Tockner B, Scharler $C$ et al (2018) Low-dose calcipotriol can elicit wound closure, anti-microbial, and anti-neoplastic effects in epidermolysis bullosa keratinocytes. Sci Rep $8: 13430$

14. Has C, Bauer JW, Bodemer Cet al (2020) Consensus reclassification of inherited epidermolysis bullosa

\section{Molecular diagnostics and treatment of epidermolysis bullosa. Multidisciplinary synchronized care in pediatric dermatology}

Epidermolysis bullosa (EB) refers to a group of genetic diseases characterized by increased fragility of epithelialized tissues with the clinical hallmark of mucocutaneous blistering after minor mechanical traumatization. A broad spectrum of cutaneous, extracutaneous and internal organ manifestations as well as primary and secondary complications cause significant morbidity and mortality and a high clinical, psychosocial and economic disease burden for patients and relatives. Although there is currently no approved curative treatment available for EB, significant advances in methodologies and techniques of molecular biology have yielded new insights into the pathogenetic processes, which boosted translational therapeutic perspectives. This is echoed by the increasing number of clinical studies in the field of EB. Current treatment approaches rely on two main strategies, i.e. corrective, potentially curative treatment approaches aiming at restoration of the defect gene or protein. In addition, diseasemodifying and symptom-relieving treatment targets secondarily induced pathogenic pathways, such as dysregulated inflammatory cascades or distinct symptoms inherent to specific subtypes, such as fibrosis, pruritus or cancer. Refined molecular diagnostics enable an early and more accurate prognosis, which facilitates genetic counseling. The management of the multisystem disease EB is complex and requires a high level of expertise and interdisciplinary coordinated care. Such resources are provided by designated centers of expertise, such as the EB Haus Austria, which as a member of the European Reference Networks for Rare Skin Diseases (ERN-Skin) guarantees clinical care, basic and clinical research activities as well as access to training and further training programs for patients, caregivers and medical specialist personnel.

\section{Keywords}

Congenital abnormalities · Blister · Inflammation · Squamous cell carcinoma · Gene therapy

and other disorders with skin fragility. BrJDermatol 183:614-627

15. Has C, Fischer J (2019) Inherited epidermolysis bullosa: new diagnostics and new clinical phenotypes. Exp Dermatol 28:1146-1152

16. Has C, Liu L, Bolling MC et al (2020) Clinical practice guidelines for laboratory diagnosis of epidermolysis bullosa. Br J Dermatol 182:574-592

17. Has C, South A, Uitto J (2020) Molecular therapeutics in development for epidermolysis bullosa: update 2020. Mol Diagn Ther 24:299-309

18. Hintner H, Stingl G, Schuler G et al (1981) Immunofluorescence mapping of antigenic determinants within the dermal-epidermal junction in the mechanobullous diseases. J Invest Dermatol 76:113-118

19. Hirsch T, Rothoeft T, Teig Netal (2017) Regeneration of the entire human epidermis using transgenic stem cells. Nature 551:327-332

20. Kocher T, March OP, Bischof J et al (2020) Predictable CRISPR/Cas9-mediated COL7A1 reframing for dystrophic epidermolysis bullosa. J Invest Dermatol 140:1985-1993.e5

21. March OP, Kocher T (2020) Context-dependent strategies for enhanced genome editing of genodermatoses. Cells 9(1):112

22. Peking P, Breitenbach JS, Ablinger $M$ et al (2019) An ex vivo RNA trans-splicing strategy to correct human generalized severe epidermolysis bullosa simplex. Br J Dermatol 180:141-148

23. Prodinger C, Bauer JW, Laimer M (2020) Translational perspectives to treat Epidermolysis bullosaWhere do we stand? Exp Dermatol 29:1112-1122

24. Prodinger C, Laimer M, Bauer JW et al (2020) EB (epidermolysis bullosa)-House Austria: pioneering work for the care of patients with rare diseases. J Dtsch Dermatol Ges 18:1229-1235

25. Saunderson RB, Vekic DA (2019) A retrospective cohort study evaluating the accuracy of clinical diagnosis compared with immunofluorescence and electron microscopy in children with inherited epidermolysis bullosa. $\mathrm{Br} J$ Dermatol 180:1258-1259

26. Tang JY, Marinkovich MP, Lucas E et al (2021) A systematic literature review of the disease burden in patients with recessive dystrophicepidermolysis bullosa. Orphanet J Rare Dis 16:175

27. Wagner RN, Piñón Hofbauer J, Wally V et al (2021) Epigenetic and metabolic regulation of epidermal homeostasis. Exp Dermatol 30(8):1009-1022

28. Wally V, Hovnanian A, Ly J et al (2018) Diacerein orphan drug development for epidermolysis bullosa simplex: a phase $2 / 3$ randomized, placebocontrolled, double-blind clinical trial. J Am Acad Dermatol 78:892-901.e7

29. Wally V, Kitzmueller S, Lagler F et al (2013) Topical diacerein for epidermolysis bullosa: a randomized controlled pilot study. Orphanet J Rare Dis 8:69

30. Wimmer M, Zauner R, Ablinger M et al (2020) A cancer stem cell-like phenotype is associated with miR-10b expression in aggressive squamous cell carcinomas. Cell Commun Signal 18:61 